TP Periodica Polytechnica Mechanical Engineering

61(3), pp. 173-183, 2017

https://doi.org/10.3311/PPme.9378

Creative Commons Attribution (i)

RESEARCH ARTICLE

\section{A Study on Solute Dispersion in a Three Layer Blood-like Liquid Flowing through a Rigid Artery}

\author{
Sudip Debnath ${ }^{1}$, Apu Kumar Saha ${ }^{1 *}$, Ashis Kumar Roy ${ }^{1}$
}

Received 26 April 2016; accepted after revision 27 October 2016

\begin{abstract}
The unsteady dispersion of a solute has been discussed by the method of generalized dispersion technique in a bloodlike liquid flowing through a pipe under the combined effects of finite yield stress and irreversible absorption into the wall. The solvent is enacted as a three-layered liquid by considering the center liquid as a Casson liquid (a core of red blood cell suspension) and a peripheral layer of plasma as a Newtonian liquid. An asymptotic representation for the convection and dispersion coefficients has been shown only for large values of time, which will not hamper the study of physical behavior of the system. The objective of the present study is to examine the nature of exchange coefficient, convective coefficient and in particular, dispersion coefficient together with mean concentration distribution under the effect of absorption parameter $(\beta)$, yield stress $\left(\tau_{y}\right)$ (equivalently the plug radius $\left(R_{p}\right)$ ) and peripheral layer variation (i.e., ratio of central core radius to normal artery radius $\left.\left(R_{d}\right)\right)$. It is found that the presence of peripheral layer makes some important increment in dispersion coefficient compared to single phase Casson liquid for small absorption. Increase in both diffusivity $\left(D^{*}\right)$ and Peclet number (Pe) make a significant decrement in the magnitude of dispersion coefficient with respect to absorption rate. The decrease in peak of the mean concentration distribution with the increase in reaction rate is found irrespective of the nature of reaction.
\end{abstract}

\section{Keywords}

Casson liquid, yield stress, Axial-dispersion coefficient, irreversible reaction

\footnotetext{
${ }^{1}$ Department of Mathematics, National Institute of Technology Agartala,

Tripura, 799046, India

*Corresponding author, e-mail: apusaha_nita@yahoo.co.in
}

\section{Introduction}

The rate of broadening of a cloud of matter in a flowing stream is due to dispersion phenomena and thus it can be utilized as an efficient means to accomplish dilution or mixing. Because of its wide applications in the arena of chemical engineering, physiological fluid dynamics, environmental sciences, bio medical engineering etc., investigations of longitudinal dispersion of a solute in an exceedingly solvent flowing through a conduit (pipe/channel) is gaining additional attention among the scientific community. Specifically, through blood flow, matters like nutrients, metabolic items, drugs and so on are transported as a consequence of diffusive and convective mechanisms in physiological systems.

The first fundamental study on dispersion was initiated by Taylor [1], discussing the dispersion of a soluble matter in a viscous liquid flowing through a circular pipe under laminar condition. He observed that the spreading of solute under the joint effects of the lateral molecular diffusion and the radial velocity distribution over the cross section is symmetrical about a point moving with the average velocity of the liquid. Using the mathematical approach of moment analysis, Aris [2] extended Taylor's theory by considering streams with lower Peclet number and found that axial diffusion would play an important role in longitudinal dispersion. Sankarasubramanian and Gill [3] developed a derivative expansion method viz. 'generalized dispersion method', for large as well as small time while comparing dispersion coefficients with time. Further more, a lot of researchers added their valuable contribution to understand dispersion process more and more precious way.

Following different dispersion techniques, a large number of studies are available in literature dealing with longitudinal dispersion in steady and unsteady flows both in Newtonian and non-Newtonian liquids (pipe/channels). Using his own particular methodology, Aris [4] first examined longitudinal dispersion by considering periodic pressure gradient as the driving force in an infinite tube. The reason for making flow unsteadiness, Bandyopadhyay and Mazumder [5] and Paul [6] considered the boundary wall movement. Using method of moments Bandyopadhyay and Mazumder [7] studied the effect 
of pulsating flow on the dispersion process in a channel and Sarkar and Jayaraman [8], Mazumder and Mondal [9] investigated the effect of wall absorption on dispersion in oscillatory flow in an annulus and explained the application of their study to a catheterized artery.

In modern days, dispersion in non-Newtonian liquids has its applications in the field of bio-chemical processing, cardiovascular system, polymer processing etc. In 1993, Sharp [10] studied the dispersion phenomena in non-Newtonians fluids (Casson, Bingham plastic and power law fluids) through conduits using Taylor-Aris dispersion model. A brief review on dispersion in power law fluids was done by Agarwal and Jayaraman [11]. Using generalized dispersion model of Sankarasubramanian and Gill [3], shear augmented unsteady dispersion of a solute in a Casson fluid flowing through a conduit was analyzed by Dash et al. [12] and discussed its application in blood flow and found that yield stress considerably affects the rate of dispersion of a fluid. In recent days, Nagarani et al. [13] discussed the longitudinal dispersion and its application in catheterized artery assuming Casson fluid model through an annulus.

In some of the above investigations, the effectiveness of longitudinal dispersion in blood flow were discussed where blood has been treated as Newtonian and non-Newtonian liquid depending on the value of shear rates. Experimental studies on blood (Scott Blair [14], Charm and Kurland [15]) with the variety of haematocrits, anticoagulants, temperature and so on and recommended that the behaviour of blood at low shear rate can be described sufficiently by the Casson model. In particular, when blood flows through small blood vessels, the presence of a peripheral layer of plasma (Newtonian liquid) and a core region of suspension of all the erythrocytes as a non-Newtinian liquid can be seen, which was experimentally shown by Bugliarello and Sevilla [16] and Cokelet [17]. The assumption of Newtonian behavior of blood is acceptable for high shear rate flow through larger arteries [18]. But, blood, being a suspension of cells in plasma, exhibits non-Newtonian behavior at low shear rate $<10 / \mathrm{s}$ in small diameter arteries $(0.02-0.1 \mathrm{~mm})$ [19]. Thus for a practical portrayal of blood stream, it is more fitting to regard blood as a two-liquid model comprising all the erythrocytes assumed to be a Casson liquid and a peripheral layer of plasma as a Newtonian liquid. There are several works available in the literature revealing the significance of the peripheral layer in the functioning of the flow characteristics in the arterial system. Srivastava and Saxena [20] showed that the resistance to flow increases as yield stress increases and also the magnitudes of the flow resistance found to be decreasing with the increase in the thickness of the peripheral layer. In a two-fluid blood flow analysis, Sankar [21] observed that both the velocity distribution and flow rate decrease whereas the wall shear, width of the plug flow region and longitudinal impedance increase when the yield stress increases. While following literature, we have seen very few works (Shukla and Parihar [22], and Shukla and Gupta [23]) of dispersion considering peripheral layer effect.

To the best of our knowledge there is hardly any work where the effect of peripheral layer is considered on dispersion process, when core region is a Casson liquid and peripheral layer of plasma is a Newtonian liquid. In addition, the present work deals with the irreversible absorption at the boundary of pipe. Also, in a significant change from previous modeling exercises in the study of hydrodynamic dispersion, we consider, different diffusivity yet constant in the three regions viz., Plug, Casson and Newtonian regions, which we think is the most suitable study for the blood-like liquid flow analysis.

\section{Mathematical formulation}

A uni-directional, steady laminar, axial, fully-developed flow of an incompressible, three-layer liquid through a circular pipe of radius $\overline{\mathrm{R}}$ is considered. It is assumed that the core region is the red blood cell suspension enclosed by a peripheral layer of plasma. The Casson model is used for characterizing the blood rheology in the core region whereas the peripheral layer of plasma follows Newtonian liquid.

Fig. 1 shows the flow geometry with a cylindrical coordinate system where the axial and radial coordinates are represented by $\bar{z}$ and $\bar{r}$ respectively (the bar denotes dimensional quantities). Once the flow is fully developed and for the low Reynolds number flow it can be shown that the radial velocity is negligibly small and can be neglected. Thus the fully developed flow is only in the axial direction hence all quantities are independent of $\theta$.

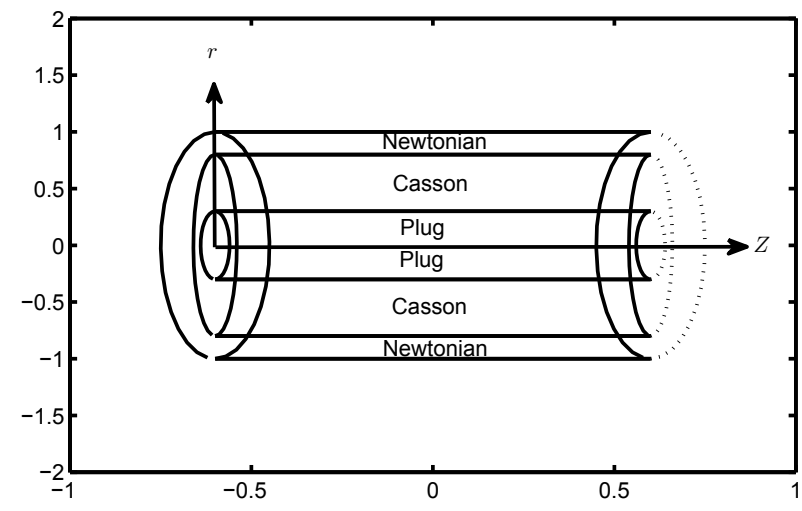

Fig. 1 Schematic diagram of the setup under consideration

We assume that Casson liquid occupies the region

$$
\mathcal{R}_{c}=\left\{(\bar{r}, \bar{z}) / 0 \leq \bar{r} \leq \bar{R}_{o},-\infty<\bar{z}<\infty\right\},
$$

and Newtonian liquid the region

$$
\mathcal{R}_{n}=\left\{(\bar{r}, \bar{z}) / \bar{R}_{o} \leq \bar{r} \leq \bar{R}-\infty<\bar{z}<\infty\right\} .
$$


Again, within the Casson liquid region, plug flow is assumed in the region:

$$
\mathcal{R}_{p}=\left\{(\bar{r}, \bar{z}) / 0 \leq \bar{r} \leq \bar{R}_{p},-\infty<\bar{z}<\infty\right\}
$$

The governing equations of motion for the axial flow are given by

Continuity equation:

$$
\frac{\partial \bar{u}}{\partial \bar{z}}=0
$$

Momentum equation:

$$
\begin{array}{cc}
\bar{\rho}_{c} \frac{\partial \bar{u}_{c}}{\partial \bar{t}}=-\frac{\partial \bar{p}}{\partial \bar{z}}-\frac{1}{\bar{r}} \frac{\partial\left(\bar{r} \bar{\tau}_{c}\right)}{\partial \bar{r}} \quad 0 \leq \bar{r} \leq \bar{R}_{o}, \\
\bar{\rho}_{n} \frac{\partial \bar{u}_{n}}{\partial \bar{t}}=-\frac{\partial \bar{p}}{\partial \bar{z}}-\frac{1}{\bar{r}} \frac{\partial\left(\bar{r} \bar{\tau}_{n}\right)}{\partial \bar{r}} \quad \bar{R}_{o} \leq \bar{r} \leq \bar{R} .
\end{array}
$$

where, $\left(-\frac{\partial \bar{p}}{\partial \bar{z}}\right)$ is the constant pressure gradient.

The shear stress in the regions $\mathcal{R}_{c}$ and $\mathcal{R}_{n}$ are given by the constitutive relations:

$$
\left.\begin{array}{l}
\bar{\tau}_{c}^{\frac{1}{2}}=\bar{\tau}_{y}^{\frac{1}{2}}+\left(-\bar{\mu}_{c} \frac{\partial \bar{u}_{c}}{\partial \bar{r}}\right)^{\frac{1}{2}}, \quad \bar{\tau}_{c} \geq \bar{\tau}_{y} \text { and } \bar{R}_{p} \leq \bar{r} \leq \bar{R}_{o}, \\
\frac{\partial \bar{u}_{c}}{\partial \bar{r}}=0, \quad \bar{\tau}_{c} \leq \bar{\tau}_{y} \text { and } 0 \leq \bar{r} \leq \bar{R}_{p}, \\
\bar{\tau}_{n}=-\bar{\mu}_{n} \frac{\partial \bar{u}_{n}}{\partial \bar{r}}, \quad \bar{R}_{o} \leq \bar{r} \leq \bar{R}
\end{array}\right\}
$$

where $\bar{\tau}_{y}$ is the yield stress. In Eq. (7) whenever $\bar{\tau}_{c} \leq \bar{\tau}_{y}$, the velocity gradient will be zero and as a consequence plug flow is seen in $\mathcal{R}_{p}$.

The boundary, symmetry and matching conditions for solving Eqs. (5)-(7) are:

$$
\left.\begin{array}{l}
\bar{\tau}_{c} \text { is finite and } \frac{\partial \bar{u}_{c}}{\partial \bar{r}}=0 \text { at } \bar{r}=0, \\
\bar{\tau}_{c}=\bar{\tau}_{n} \text { and } \bar{u}_{c}=\bar{u}_{n} \text { at } \bar{r}=\bar{R}_{o}, \\
\bar{u}_{n}=0 \text { at } \bar{r}=\bar{R},
\end{array}\right\} .
$$

Let us now consider the transport and spreading of a chemical species with the blood-like liquid flowing through the tube (rigid artery) which is supposed to be completely miscible with the liquid. The species are supposed to involve irreversible absorption into the wall.

In the present scenario the transport equation that governs $\bar{C}(\bar{t}, \bar{r}, \bar{z})$ is the unsteady, convective diffusion equation:

$$
\frac{\partial \bar{C}}{\partial \bar{t}}+\bar{u}(\bar{r}) \frac{\partial \bar{C}}{\partial \bar{z}}=\frac{\bar{D}}{\bar{r}} \frac{\partial}{\partial \bar{r}}\left(\bar{r} \frac{\partial \bar{C}}{\partial \bar{r}}\right)+\bar{D} \frac{\partial^{2} \bar{C}}{\partial \bar{z}^{2}}
$$

where $\bar{D}$ is the constant molecular diffusivity and considered to be different in the three regions $\mathcal{R}_{p}, \mathcal{R}_{c}$ and $\mathcal{R}_{n}$. So we assume

$$
\bar{D}=\left\{\begin{array}{ll}
\bar{D}_{p} & \text { in } \mathcal{R}_{p} \\
\bar{D}_{c} & \text { in } \mathcal{R}_{c} \\
\bar{D}_{n} & \text { in } \mathcal{R}_{n}
\end{array}\right\}
$$

The initial and boundary conditions for the transport Eq. (9) are considered as

$$
\bar{C}(0, \bar{r}, \bar{z})=\bar{C}_{0} B(\bar{r}) \psi(\bar{z}), \quad 0<\bar{r}<\bar{R}
$$

$$
\frac{\partial \bar{C}}{\partial \bar{r}}=0 \quad \text { at } \quad \bar{r}=0
$$

$$
\frac{\partial \bar{C}}{\partial \bar{r}}+\bar{\beta} \bar{C}=0 \quad \text { at } \quad \bar{r}=\bar{R},
$$

where $B(\bar{r})$ and $\psi(\bar{z})$ are needed to be specified to solve Eq. (9), $\bar{C}_{0}$ is the initial concentration of slug input. The absorbing boundary condition at the wall of the tube is represented by Eq. (13), where $\bar{\beta}$ is the irreversible absorption parameter.

Using the following dimensionless quantities

$$
\begin{aligned}
& \left.\begin{array}{l}
t=\frac{\bar{D}_{n} \bar{t}}{\bar{R}^{2}}, \quad r=\frac{\bar{r}}{\bar{R}}, \quad z=\frac{\bar{D}_{n} \bar{z}}{\bar{R}^{2} u_{0}}, \quad u_{0}=-\frac{\bar{R}^{2}}{4 \bar{\mu}_{n}} \frac{d \bar{p}}{d \bar{z}}, \\
u_{c}=\frac{\bar{u}_{c}}{u_{0}}, \quad u_{n}=\frac{\bar{u}_{n}}{u_{0}}, C=\frac{\bar{C}}{\bar{C}_{0}}, \quad \tau_{c}=\frac{\bar{\tau}_{n}}{\bar{\mu}_{n}\left(\frac{u_{0}}{\bar{R}}\right)}, \\
\tau_{n}=\frac{\bar{\tau}_{n}}{\bar{\mu}_{n}\left(\frac{u_{0}}{\bar{R}}\right)}, \quad \tau_{y}=\frac{\bar{\tau}_{y}}{\bar{\mu}_{n}\left(\frac{u_{0}}{\bar{R}}\right)}, \quad R_{0}=\frac{\bar{R}_{o}}{\bar{R}}, \\
R_{p}=\frac{\bar{R}_{p}}{\bar{R}}, \quad \beta=\bar{\beta} \bar{R}, \quad D^{*}=\frac{\bar{D}}{\bar{D}_{n}}, \quad P e=\frac{u_{0} \bar{R}}{\bar{D}_{n}}
\end{array}\right\}
\end{aligned}
$$

the above system of Eqs. (9)-(13) can be written in dimensionless form as:

$$
\begin{aligned}
\frac{\partial C}{\partial t}+u(r) \frac{\partial C}{\partial z}= & \frac{D^{*}}{r} \frac{\partial}{\partial r}\left(r \frac{\partial C}{\partial r}\right)+\frac{D^{*}}{P e^{2}} \frac{\partial^{2} C}{\partial z^{2}}, \\
D^{*} & =\left\{\begin{array}{ll}
D_{p} & \text { in } \mathcal{R}_{p} \\
D_{c} & \text { in } \mathcal{R}_{c} \\
1 & \text { in } \mathcal{R}_{n}
\end{array}\right\}
\end{aligned}
$$

along with initial and boundary conditions

$$
\begin{gathered}
C(0, r, z)=B(r) \psi(z), \quad(0<r<1) \\
\frac{\partial C}{\partial r}=0 \quad \text { at } \quad r=0, \\
\frac{\partial C}{\partial r}=-\beta C \quad \text { at } \quad r=1,
\end{gathered}
$$

Here $u_{0}$ is the time averaged axial velocity and $\beta$ is the absorption parameter or first order reaction rate representing the rate of loss on the wall. $P e$ is the Peclet number which measures the relative characteristic time of the diffusion process $\left(\frac{R^{2}}{D}\right)$ to the convection process $\left(\frac{R}{u_{0}}\right)$.

\section{Velocity distribution}

With the help of above boundary conditions (8) in dimensionless form, the velocity distribution is given by Sankar and Lee [24]. 


$$
\begin{aligned}
& u_{c}(r)=\left[1-R_{o}^{2}+R_{o}^{2}\left\{1-\left(\frac{r}{R_{o}}\right)^{2}-\frac{4 \sqrt{2}}{3} \sqrt{\frac{\tau_{y}}{R_{o}}}\right.\right. \\
& \left.\left(1-\left(\frac{r}{R_{o}}\right)^{\frac{3}{2}}\right)+\frac{\tau_{y}}{R_{o}}\left(1-\frac{r}{R_{o}}\right)\right\} R_{p} \leq r \leq R_{o}, \\
& u_{p}=\left[1-R_{o}^{2}+R_{o}^{2}\left\{1-\left(\frac{R_{p}}{R_{o}}\right)^{2}-\frac{4 \sqrt{2}}{3} \sqrt{\frac{\tau_{y}}{R_{o}}}\right.\right. \\
& \left.\left.\left(1-\left(\frac{R_{p}}{R_{o}}\right)^{\frac{3}{2}}\right)+\frac{\tau_{y}}{R_{o}}\left(1-\frac{R_{p}}{R_{o}}\right)\right\}\right] 0 \leq r \leq R_{p}, \\
& u_{n}(r)=\left[1-r^{2}\right] \quad R_{o} \leq r \leq 1,
\end{aligned}
$$

Where $u_{c}$ and $u_{n}$ are the velocities for shear flow in Casson and Newtonian regions. The constant velocity $u_{p}$ appears only in plug flow region having radius $R_{p}=\frac{\tau_{y}}{2}$. Also $R_{o}(\leq 1)$ is the ratio of the central core radius to the normal pipe radius.

\section{Generalized dispersion model}

According to and Sankarasubramanian and Gill [3], the expansion of concentration $C$ subject to axial derivatives of mean concentration can be written as

$$
C(r, z, t)=\sum_{i=0}^{\infty} f_{i}(r, t) \frac{\partial^{i} C_{m}}{\partial z^{i}},
$$

where $f_{k}$ 's are the time-dependent coefficients to be determined and the dimensionless area-average concentration $C_{m}$ is given by

$$
C_{m}=C_{m}(z, t)=\frac{\int_{0}^{2 \pi} d \theta \int_{0}^{1} r C d r}{\int_{0}^{2 \pi} d \theta \int_{0}^{1} r d r}=2 \int_{0}^{1} r C d r,
$$

Using Eq. (22) in Eq. (15) along with Eq. (21) and also the boundary conditions (18) and (19), the following dispersion model for $C_{m}$ gives

$$
\frac{\partial C_{m}}{\partial t}=\sum_{i=0}^{\infty} M_{i}(t) \frac{\partial^{i} C_{m}}{\partial z^{i}},
$$

According to Sankarasubramanian and Gill [3], it will be sufficient to truncate the infinite sum of Eq. (23) after first three terms, i.e.

$$
\frac{\partial C_{m}}{\partial t}=M_{0}(t)+M_{1}(t) \frac{\partial C_{m}}{\partial z}+M_{2}(t) \frac{\partial^{2} C_{m}}{\partial z^{2}},
$$

Where

$$
\begin{gathered}
M_{0}(t)=-2 \beta D^{*} f_{0}(1, t), \\
M_{1}(t)=-2 \beta D^{*} f_{1}(1, t)-2 \int_{0}^{1} u(r) r f_{0}(r, t) d r, \\
M_{2}(t)=\frac{D^{*}}{P e^{2}}-2 \beta D^{*} f_{2}(1, t)-2 \int_{0}^{1} u(r) r f_{1}(r, t) d r,
\end{gathered}
$$

Because of the non-zero solute flux at the outer boundary of wall, $M_{0}(t)$ arises, which corresponds to the absorption parameter. As the solute undergoes with irreversible reaction at the outer boundary it will deplete with time that makes negative exchange coefficient $\left(M_{0}(t)\right)$. In such a case, exchange coefficient would be positive as $\beta$ in the Eq. (19) has been considered with negative sign. $M_{1}(t)$ and $M_{2}(t)$ correspond to the convective and dispersion coefficients respectively. It is necessary to mention here that $M_{2}(t)$ is analogous to the dispersion coefficient $\left(D_{a}\right)$ obtained from Aris 'Moment method'.

To obtain the above coefficients one needs to find the corresponding functions $f_{k}$ 's $(\mathrm{k}=0,1,2)$. So, Eq. (21) is used in Eq. (15) along with Eq. (23) and equating the coefficient of $\frac{\partial^{k} C_{m}}{\partial z^{k}}$, the resulting partial differential equations are

$$
\begin{aligned}
& \frac{\partial f_{k}}{\partial t}+\sum_{i=0}^{k} M_{i}(t) f_{k-i}(r, t)+u(r) f_{k-1}-\frac{D^{*}}{r} \frac{\partial}{\partial r}\left(r \frac{\partial f_{k}}{\partial r}\right) \\
& -\frac{D^{*}}{P e^{2}} f_{k-2}=0,
\end{aligned}
$$

where $f_{-1}=f_{-2}=0$ and $k=0,1,2$

From Eq. (17)-(19), the initial conditions for $C_{m}$ and $f_{k}$ are

$$
\begin{gathered}
C_{m}(z, 0)=2 \psi(z) \int_{0}^{1} B(r) r d r, \\
f_{k}(r, 0)= \begin{cases}1 & \text { for } k=0 \\
0 & \text { for } k=1,2\end{cases}
\end{gathered}
$$

and the boundary conditions for the pipe are

$$
\begin{gathered}
\frac{\partial f_{k}}{\partial r}=0 \quad \text { at } r=0 \text { for } k=0,1,2 . \\
\frac{\partial f_{k}}{\partial r}=-\beta f_{k}(r, t) \text { at } r=1 \text { for } k=0,1,2 .
\end{gathered}
$$

also

$$
C_{m}(t, \infty)=\frac{\partial c_{m}}{\partial z}(t, \infty)=0
$$

and

$$
\int_{0}^{1} f_{k}(t, r) r d r=\frac{1}{2} \delta_{k 0} \quad \text { for } k=0,1,2
$$

\subsection{Estimation of Exchange Coefficient $\left(M_{0}\right)$}

The solution for $f_{0}(t, r)$ that satisfies the Eq. (27), (29) and (31) can be obtained from Eq. (25) as

$$
f_{0}(t, r)=\frac{\sum_{0}^{\infty} A_{n} J_{0}\left(\frac{\mu_{n} r}{D^{*}}\right) e^{-\mu_{n}^{2} t}}{2 \sum_{0}^{\infty}\left(\frac{A_{n} D^{*}}{\mu_{n}}\right) J_{1}\left(\frac{\mu_{n}}{D^{*}}\right) e^{-\mu_{n}^{2} t},}
$$

Where $J_{0}$ and $J_{1}$ are the Bessel functions of the first kind of order zero and one respectively and $A_{n}$ 's are constants that can be calculated from the initial conditions using the concept of orthogonality of the eigen functions $J_{0}\left(\mu_{n} r\right)$. The eigen values $\mu_{n}$ 's are the root of the transcendental equation

$$
\mu_{n} J_{1}\left(\frac{\mu_{n}}{D^{*}}\right)-\beta D^{*} J_{0}\left(\frac{\mu_{n}}{D^{*}}\right)=0 \quad \mathrm{n}=0,1,2,3, \ldots
$$


Further on using Eq. (24a), the exchange coefficient can be obtained as

$$
M_{0}(t)=-\frac{\sum_{0}^{\infty} \frac{A_{n} \mu_{n}}{D^{*}} J_{1}\left(\frac{\mu_{n} r}{D^{*}}\right) e^{-\mu_{n}^{2} t}}{\sum_{0}^{\infty}\left(\frac{A_{n} D^{*}}{\mu_{n}}\right) J_{1}\left(\frac{\mu_{n}}{D^{*}}\right) e^{-\mu_{n}^{2} t},}
$$

From Eq. (34), it is clear that $M_{0}(t)$ does not depend on velocity distribution though it depends on the initial solute distribution.

\subsection{Estimation of Convection Coefficient $\left(M_{1}\right)$}

The coupling connection between Eq. (24b), (24c) and Eq. (25) ensures that the outcomes for the transformed problem, say $f_{1}(t, r)$ and $M_{1}(t)$ is a complicated one. Even $f_{2}(t, r)$ and $M_{2}(t)$ are more difficult to solve. Therefore, keeping physical insights in mind the asymptotic steady-state representations of $f_{k}(t, r)$ and $M_{k}(t)$ for the case of steady flow are considered in this work.

The asymptotic representation for $f_{0}$ and $M_{0}$, can be obtained from Eq. (32) and Eq. (34), as $t \rightarrow \infty$

$$
\begin{gathered}
f_{0}(\infty, r)=\frac{\mu_{0}}{2 D^{*} J_{1}\left(\frac{\mu_{0}}{D^{*}}\right)} J_{0}\left(\frac{\mu_{0} r}{D^{*}}\right), \\
M_{0}(\infty)=-\left(\frac{\mu_{0}}{D^{*}}\right)^{2},
\end{gathered}
$$

where $\mu_{0}$ is the first root of the transcendental equation (33) with the smallest magnitude.

Due to large values of time the steady state function $f_{k}(r)$ will satisfy the equation

$$
\begin{aligned}
& \frac{D^{*}}{r} \frac{d}{d r}\left(r \frac{d f_{k}}{d r}\right)+\mu_{0}^{2} f_{k}=u(r) f_{k-1}-\frac{D^{*}}{P e^{2}} f_{k-2} \\
& +\sum_{i=1}^{k} M_{i} f_{k-i} \quad \text { for } k=1,2 .
\end{aligned}
$$

The boundary conditions on $f_{k}(r)$ are

$$
\begin{gathered}
f_{k}(0)=\text { finite, } \\
\frac{\partial f_{k}(1)}{\partial r}=-\beta f_{k}(1) \text { for } k=1,2 .
\end{gathered}
$$

also

$$
\int_{0}^{1} f_{k}(r) r d r=0 \text { for } k=1,2 .
$$

Again Eq. (24b) and Eq. (24c) reduces to

$$
\begin{gathered}
M_{1}=-2 \beta D^{*} f_{1}(1)-2 \int_{0}^{1} u(r) r f_{0}(r) d r, \\
M_{2}=\frac{D^{*}}{P e^{2}}-2 \beta D^{*} f_{2}(1)-2 \int_{0}^{1} u(r) r f_{1}(r) d r,
\end{gathered}
$$

Since Eq. (37) and Eq. (41a), (41b) are coupled, subsequently an option representation for $M_{k}$ regarding known function $f_{i}$ and the known $M_{i}(\mathrm{i}=1,2)$ is coveted. The use of the solvability condition in Eq. (37) gives the expression for $M_{k}$ as

$$
M_{k}=\frac{\int_{0}^{1} r J_{0}\left(\frac{\mu_{0} r}{D^{*}}\right)\left\{\frac{D^{*}}{P e^{2}} f_{k-2}(r)-u(r) f_{k-1}(r)-\sum_{i=1}^{k-1} M_{i} f_{k-i}(r)\right\} d r}{\int_{0}^{1} r f_{0}(r) J_{0}\left(\frac{\mu_{0} r}{D^{*}}\right) d r} ;
$$

for $k=1,2$.

Thus the form of convection coefficient $M_{1}$ obtained from Eq. (42) and Eq. (33) is:

$$
M_{1}=\frac{-2 \mu_{0}^{2}}{\left(\mu_{0}^{2}+\left(D^{*} \beta\right)^{2}\right) J_{0}^{2}\left(\frac{\mu_{0}}{D^{*}}\right)} \int_{0}^{1} u(r) r J_{0}^{2}\left(\frac{\mu_{0} r}{D^{*}}\right) d r,
$$

\subsection{Estimation of Dispersion Coefficient $\left(M_{2}\right)$}

Now, using Eq. (43) in Eq. (37) the solution for $f_{1}(r)$ satisfying the boundary conditons (38), (39) and (40), we have

$$
f_{1}(r)=\sum_{0}^{\infty} B_{n} J_{0}\left(\frac{\mu_{n} r}{D^{*}}\right)
$$

where

$$
B_{0}=\frac{-\mu_{0}}{J_{1}\left(\frac{\mu_{0}}{D^{*}}\right)} \sum_{n=1}^{\infty} B_{n} \frac{J_{1}\left(\frac{\mu_{n}}{D^{*}}\right)}{\mu_{n}}
$$

Combining both the Eq. (44) and Eq. (45) we get

$$
f_{1}=\sum_{n=1}^{\infty} B_{n}\left[J_{0}\left(\frac{\mu_{n} r}{D^{*}}\right)-\frac{\mu_{0}}{J_{1}\left(\frac{\mu_{0}}{D^{*}}\right)} \frac{J_{1}\left(\frac{\mu_{n}}{D^{*}}\right)}{\mu_{n}} J_{0}\left(\frac{\mu_{0} r}{D^{*}}\right)\right],
$$

where $B_{n}$ 's are given by

$$
B_{n}=\frac{2 D^{*} \mu_{n}^{2} \int_{0}^{1}\left(u(r)+M_{1}\right) f_{0}(r) r J_{0}\left(\frac{\mu_{n} r}{D^{*}}\right) d r}{\left(\mu_{0}^{2}-\mu_{n}^{2}\right)\left(\mu_{n}^{2}+\left(D^{*} \beta\right)^{2}\right) J_{0}^{2}\left(\frac{\mu_{n}}{D^{*}}\right)},
$$

Now using Eq. (47) in Eq. (46) and then in Eq. (42), we have the dispersion coefficient as

$$
M_{2}=\frac{D^{*}}{P e^{2}}-\frac{4 \mu_{0} D^{*} J_{1}\left(\frac{\mu_{0}}{D^{*}}\right) \int_{0}^{1}\left(u(r)+M_{1}\right) f_{1}(r) r J_{0}\left(\frac{\mu_{0} r}{D^{*}}\right) d r}{\left(\mu_{0}^{2}+\left(D^{*} \beta\right)^{2}\right) J_{0}^{2}\left(\frac{\mu_{0}}{D^{*}}\right)},
$$

\section{Distributions of mean concentration}

On truncating the terms beyond $M_{2}(t)$, the solution of the Eq. (23) with initial and boundary conditions (26)-(30) is given by

$$
C_{m}(t, z)=\frac{1}{2 P e \sqrt{\pi \xi}} \exp \left(\zeta-\frac{z_{1}^{2}}{4 \xi}\right)
$$

where

$$
\begin{gathered}
\zeta(t)=\int_{0}^{t} M_{0}(\eta) d \eta, \\
z_{1}(t, z)=z+\int_{0}^{t} M_{1}(\eta) d \eta, \\
\xi(t)=\int_{0}^{t} M_{2}(\eta) d \eta,
\end{gathered}
$$



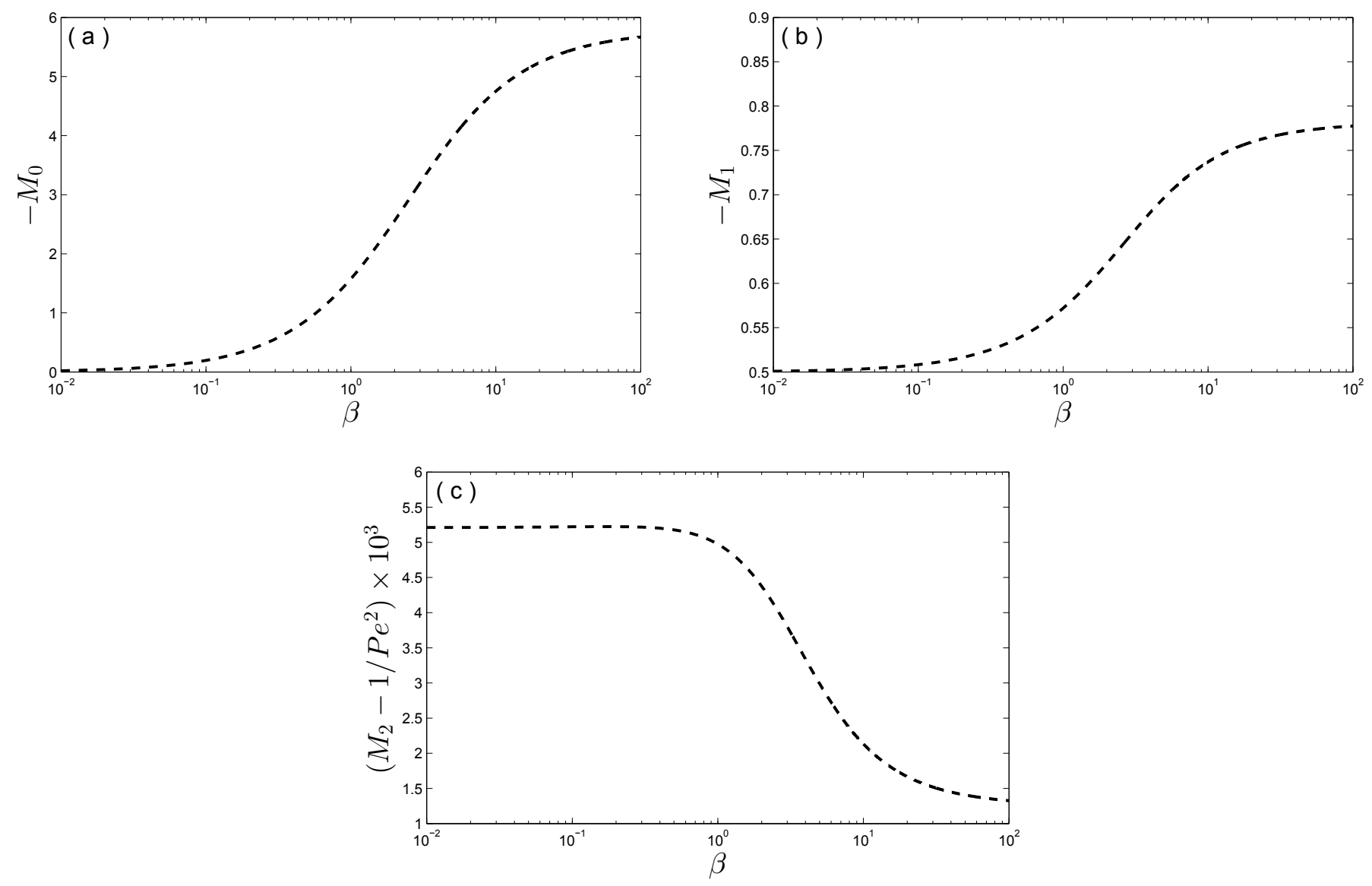

Fig. 2 Variation of the (a) Negative exchange coefficient; (b) Negative convection coefficient and (c) Dispersion coefficient with respect to $\beta$; when $R_{p}=0.0, R_{\mathrm{o}}=1.0, D_{p}=D_{c}=1.0$ and $P e=100$.

For large values of time, the coefficients $M_{0}, M_{1}$ and $M_{2}$ in Eq. (50) represents their asymptotic values. Also, as the asymptotic values of $M_{0}, M_{1}$ and $M_{2}$ [obtained from Eq. (36), Eq. (43) and Eq. (48)] are not the functions of radial non-uniformities in the initial distribution, hence it has no effect in the results of Eq. (49).

\section{Results and Discussion}

The present analysis mainly deals with the dispersion of a solute in a blood-like liquid flowing through a narrow pipe under the effect of peripheral layer variation and irreversible absorption into the wall. In a very particular way the present study tries to simulate mathematically the behaviour of the flowing liquid as blood-like liquid flow. It was already discussed that the unsteady dispersion coefficient has to be analyzed by generalized dispersion technique, so the entire process is based on three effective transport coefficient, viz. exchange coefficient $\left(M_{0}\right)$, convection coefficient $\left(M_{1}\right)$ and dispersion coefficient $\left(M_{2}\right)$. As we have experienced some difficulties to present the expressions relating $f_{k}$ 's and $M_{k}$ 's $(k \geq 1)$, we focus on asymptotic values of these coefficients for a large values of time, though it will not affect the physics of the system. It is observed from the asymptotic formulation that the exchange coefficient does not depend on the finite yield stress of the fluid, but the other two coefficients depend on yield stress or plug radius. Again the absorption parameter $\beta$ has an important role on all three coefficients in their asymptomatic state.

A MATLAB code is developed for estimating the coupling effect of different parameters and the integrations appear in the expressions are all calculated by Simpson's 1/3 rule. To verify the accuracy of our procedure of handling, we have successfully tested few of our results with some existing results, viz. when $R_{o}=1, R_{p}=0$ and $D_{p}=D_{c}=1$, the asymptotic value of all three transport coefficients are totally agreed with Sankarasubramanian and Gill [3] which are shown in Fig. 2. To investigate the coupled effect of the irreversible absorption $(\beta)$, peripheral layer variation $\left(R_{o}\right)$ and yield stress $\left(\tau_{y}\right)$ or plug core radius $\left(R_{p}\right)$ on $M_{1}$ and $M_{2}$, figures have been drawn using practically significant values of the parameters which ensures the character of the flowing liquid as blood-like liquid flow. 

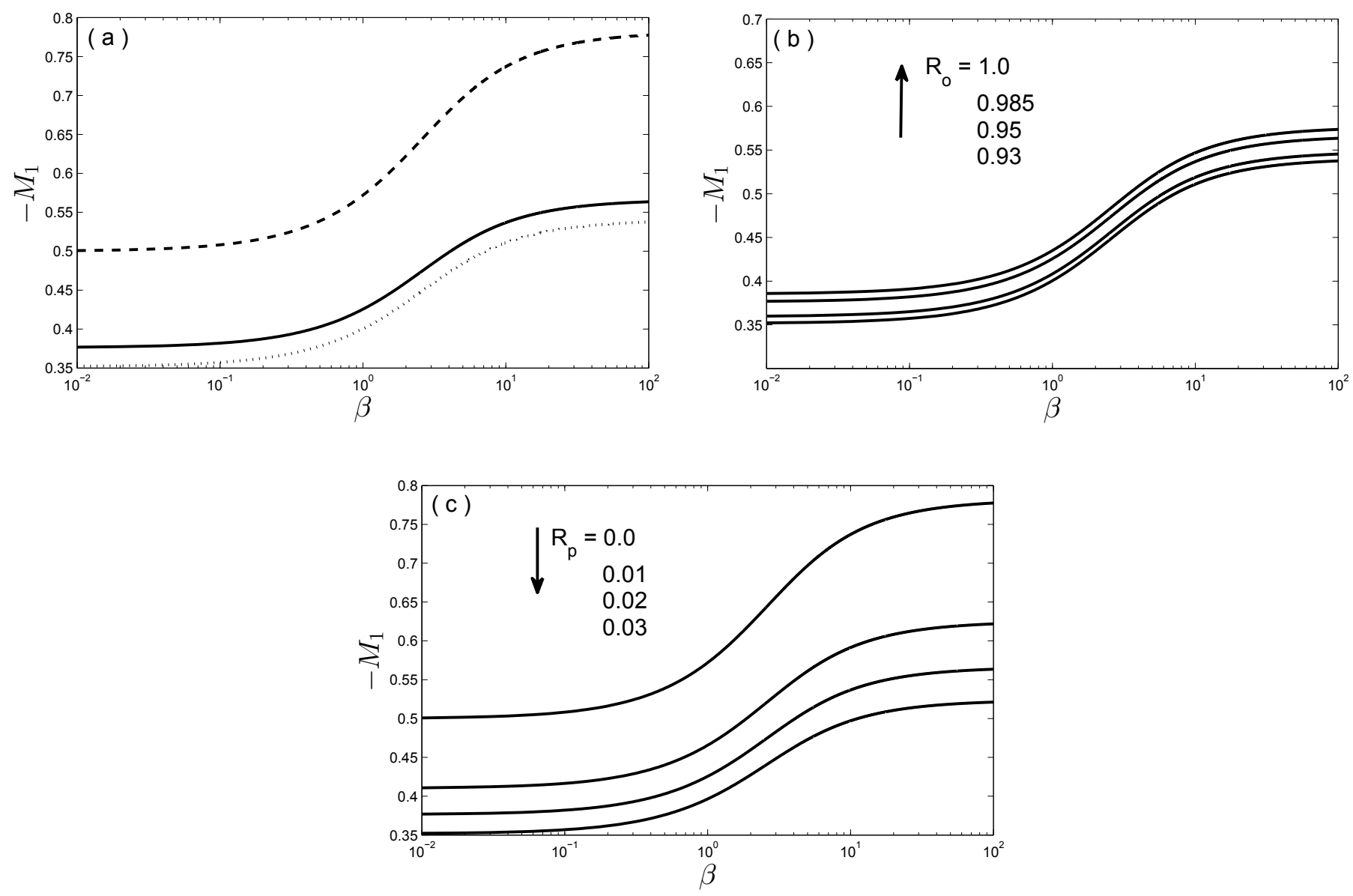

Fig. 3 Plots of asymptotic negative convective coefficient against $\beta$ for fixed $D_{p}=D_{c}=1$ and $P e=100$, (a) Newtonian liquid represented by dished line when $R_{p}=0$ and $R_{\mathrm{o}}=1$, two-layer flow is given by solid line when $R_{p}=0.02, R_{\mathrm{o}}=0.95$ and the single phase casson liquid is represented by dotted line when $R_{p}=$ 0.02 and $R_{\mathrm{o}}=1$ (b) when $R_{p}=0.02$ (c) and $R_{\mathrm{o}}=0.95$.

For example, absorption parameter $(\beta)$ is taken in the range from 0.01 to 100 to account for small to large absorption; the range for $R_{o}$ is 0.92 to 1 ; plug flow radius $\left(R_{p}\right)$ varying from 0.0 to 0.03 etc.

From Eq. (24a) it is clear that, only absorption parameter is the reason for the appearance of exchange coefficient. Hence it can be realized easily that $\left(-M_{0}\right)$ is independent of both yield stress $\left(\tau_{y}\right)$ and peripheral layer variation $\left(R_{o}\right)$.

Fig. 3 shows the variation of negative convection coefficient with absorption parameter in the presence of peripheral layer. It can be clearly seen from Fig. 3(a) that asymptotic absolute value of $M_{1}$ steadily increases as absorption increases, for a fixed $R_{o}=0.95$ and $R_{p}=0.02$. In this case when $\beta=100, M_{1}$ has increased by 1.50 times the value corresponding to $\beta=0.01$, whereas for the case of single phase Casson model and Newtonian model this increments are 1.53 and 1.55 times respectively. The reason behind the increment is the rapid enhancement in $\beta$, so the reaction at the wall consumes material in a quick manner and hence the solute moves towards the faster moving core region. Also from Fig. 3(b) it is found that the negative convective coefficient increases with $\beta$ when the thickness of peripheral layer increases. Due to the lower value of $R_{o}$ will lead to greater region of Newtonian liquid and hence larger value of the convection coefficient. Fig. 3(c) shows the variation of negative convective coefficient vs absorption parameter for different central core region with fixed $R_{o}=0.95$, the negative convective coefficient decreases with $R_{p}$ and increases with $\beta$, which completely satisfies the physical behavior of the system, since the increase of $R_{p}$ results the decrease in corresponding velocities.

From the investigation in Fig. 3, we see that, for large time, when $R_{p}=0.02$ and $R_{o}=0.95$ and $\beta$ ranges from [0.01 100], the value of $-M_{1}$ increases in the range of [0.02 - 0.03] in comparing the corresponding values of Casson model say $R_{p}=0.02, R_{o}=1$. Though $-M_{1}$ decreased in the range from [0.21 - 0.12] as collating the values of corresponding Newtonian flow, say $R_{p}=0$ and $R_{o}=1$.

Fig. 4 demonstrates the functional relationship between dispersion coefficient $\left(M_{2}\right)$ [from that the additive contribution of axial diffusion, $\frac{1}{P e^{2}}$ has been withdrawn] and $\beta$. From 

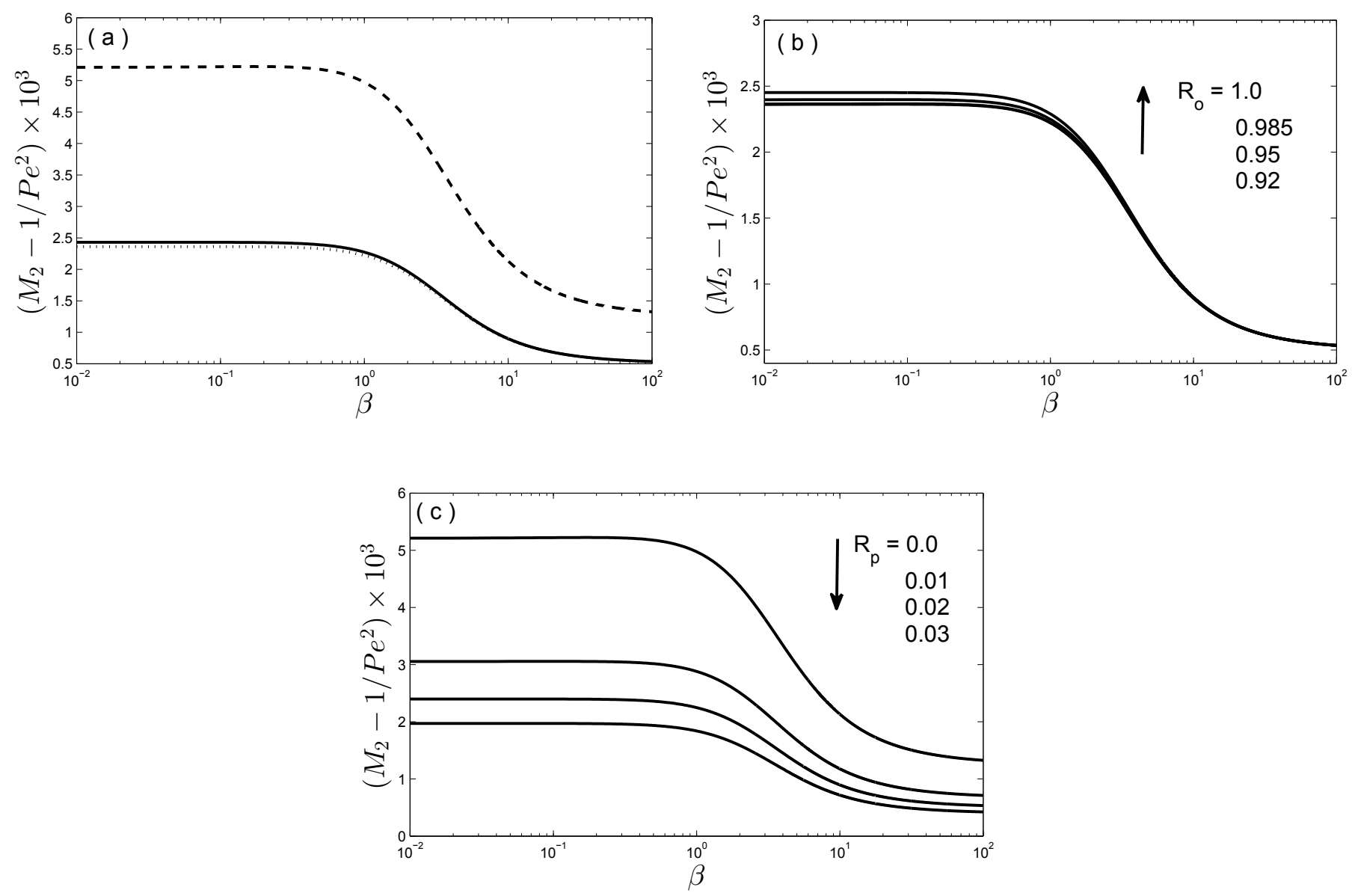

Fig. 4 Plots of asymptotic dispersion coefficient against $\beta$ for fixed $D_{p}=D_{c}=1$ and $P e=100$, (a) Newtonian liquid represented by dished line when $R_{p}=0$, and $R_{\mathrm{o}}=1$, two-layer flow is given by solid line when $R_{p}=0.02$, and $R_{o}=0.93$ and the single phase casson liquid is represented by dotted line when $R_{p}=0.02$, and $R_{o}=1$ (b) when $R_{p}=0.02$ (c) $R_{o}=0.95$.

Fig. 4(a), it has been seen that the dispersion coefficient significantly decreases as absorption increases. On the basis of physical perception, the increase of $\beta$ leads to an increase in the number of moles of reactive material undergoing chemical reaction or absorption resulting in a change in the concentration distribution across the tube and hence there is a drop in dispersion coefficient. The most remarkable thing is the effect of peripheral layer, which makes some increment of $M_{2}$ in comparison to the corresponding values of Casson model alone. The physical explanation for this increment in initial reaction domain is the interaction between $\beta$ and the thin-Newtonian region at the tube boundary, though as $\beta$ increases $M_{2}$ nearly approaches to the corresponding values in Casson model, but eventually they do not overlap. It is observed that when $\beta=100, M_{2}$ has been decreased by 0.22 times the values corresponding to $\beta=0.01$ , but for Casson and Newtonian models the decrements are found to be 0.23 and 0.25 times respectively. From Fig. 4(b) it is clear that, as $R_{o}$ decreases (or peripheral layer thickness increases) the value of dispersion coefficient has a significant increment for small absorption rate, whereas for large $\beta$ , the values are close to Casson state. Because of the presence of Newtonian layer at the boundary having large velocity as compare to the Casson layer and also due to the initial reaction with the solute, dispersion coefficient increased for weak reaction, which completely indicates the effect of peripheral layer on dispersion process. Also from Fig. 4(c), it is clear that the dispersion coefficient decreases significantly with the increase in the values of $R_{p}$. Due to increase in yield stress or plug core radius of the liquid, flow velocity decreases and hence the solute dispersion decreases.

From the above investigation in Fig. 4, we can conclude that for a fixed $R_{p}=0.02$ and $R_{o}=0.95$, asymptotic dispersion coefficient decreases in the range [2.81 - 0.80], in comparison with the corresponding values in Newtonian liquid. Though, for small and large $\beta$ these values ranging from [0.0358 $0.0002]$, following corresponding values of Casson model, say $R_{p}=0.02$ and $R_{o}=1$.

The molecular diffusivity or diffusion coefficient $\left(D^{*}\right)$ is a kinematic property of the fluid which implies that the mass of the substance diffuses through a unit surface in a unit time at a concentration gradient of unity. So, diffusion coefficient represents the spreading process, the larger values of $D^{*}$ indicates the rapid spreading of the tracer curve whereas smaller $D^{*}$ means slow spreading of solute in the liquid. 

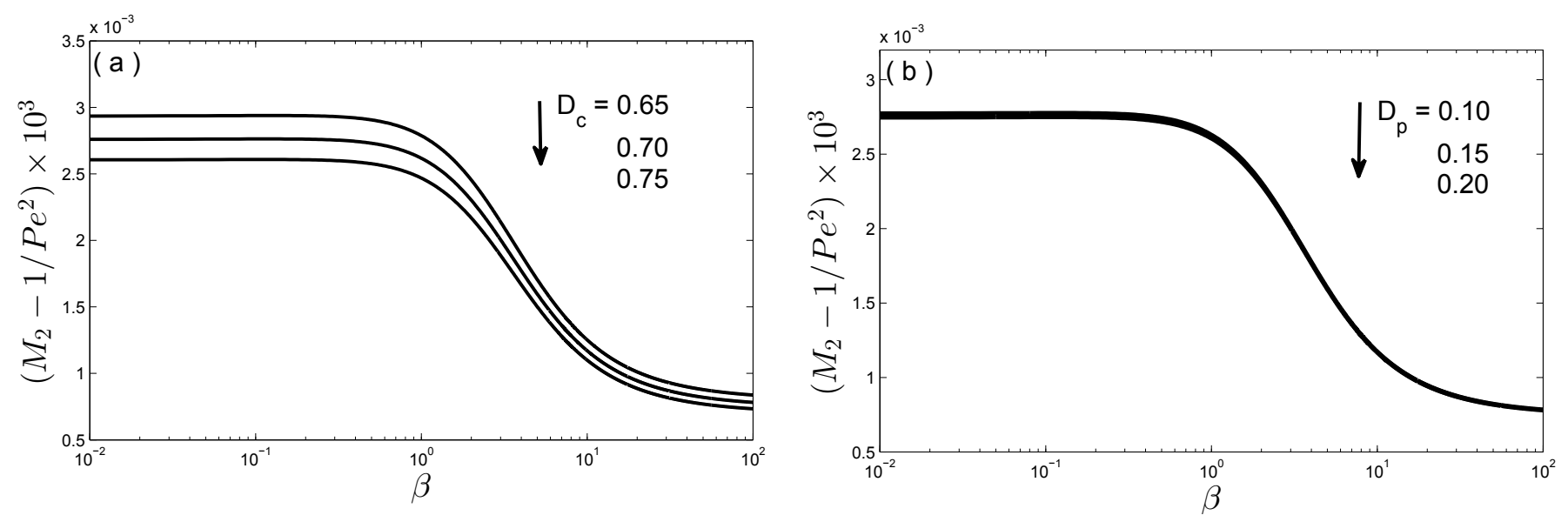

Fig. 5 Plots of asymptotic dispersion coefficient against $\beta$ for fixed $R_{o}=0.95, R_{p}=0.02$ and $P e=100$, (a) with various values of $D_{c}$ (b) with various values of $D_{p}$.

Fig. 5 shows the variation of dispersion coefficient with respect to absorption for various values of $D^{*}$. It can be depicted form Fig. 5 (a, b), for both the regions viz., plug $\left(\mathcal{R}_{p}\right)$ and Casson $\left(\mathcal{R}_{c}\right)$, large values of $D_{p}$ and $D_{c}$ decrease the magnitude the solute dispersion coefficient and reaches to its steady value when absorption is so high. The reason for this decrements is due to fast spreading of molar flux which completely agrees the physics of the system.

The convective characteristic scales are used for the dimensionless formulation that incorporates the Peclet number $(\mathrm{Pe})$ into the convection diffusion equation as a measure for fluid dispersion. Higher values of Peclet number means the effects of convection exceed those of diffusion in determining the overall mass flux, which could be the reason for small magnitude in dispersion coefficient. Fig. 6 shows that dispersion coefficient decreases as Peclet number increases.

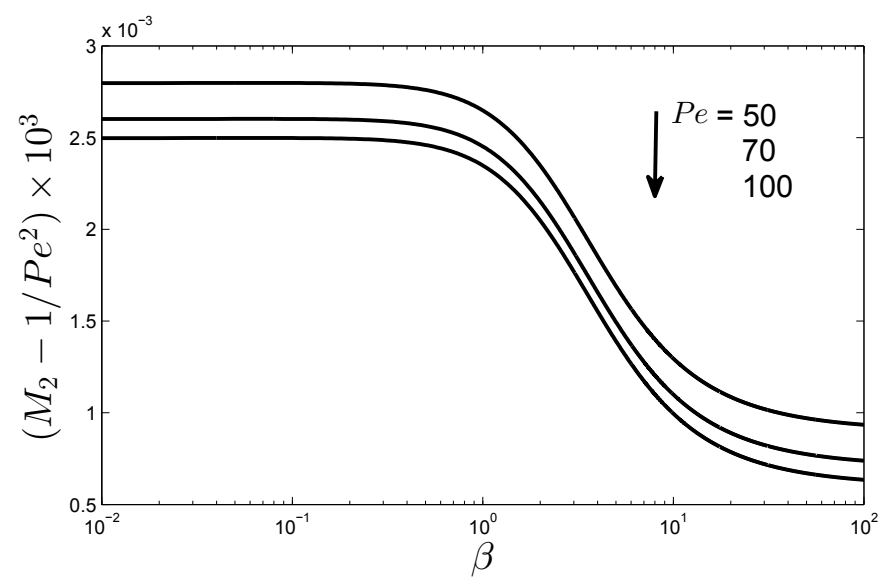

Fig. 6 Plots of asymptotic dispersion coefficient against $\beta$ for fixed $R_{o}=0.95$, $R_{p}=0.02, D_{c}=D_{p}=1$ and $P_{e}=100$, with various values of Peclet number $(P e)$.

Using the Eq. (49) and Eq. (50a), (50b), (50c), the axial variation of mean concentration distribution $\left(C_{m} \times P e\right)$ has been presented in Fig. 7 against dimensionless time $(t)$ at $z=0.5$, for different values of plug core radius $\left(R_{p}\right)$, absorption parameter $(\beta)$ and peripheral layer variation $\left(R_{o}\right)$. It is seen from the figure that, due to constant depletion occurring at the tube wall as the dispersion time $t$ increases, the peak of mean concentration decreases with $\beta$ and it may becomes falter at large time. Hence for large absorption, the dispersion coefficient may not have practical importance. From Fig. 7(c), it is observed that, when $R_{p}=0, \beta=0.01$ and $R_{o}=1$ (i.e. for Newtonian liquid model), the peak concentration is about 3.82 and when $R_{p}=0.02, \beta=0.01$ and $R_{o}=1$ (i.e. for single phase Casson model) this value is 4.66 , and finally, when $R_{p}=0.02$, $\beta=0.01$ and $R_{o}=0.95$ (i.e. for two-phase Casson model) this value is 4.79 .

\section{Conclusions}

From the aforementioned discussion, it can be said that the utility of the upgraded mathematical model explained in this paper has a significance in the study of solute dispersion. A qualitative analysis for scalar dispersion has been studied by evaluating the values of all the three coefficients using analytic approach of Sankarasubramanian and Gill [3] for large time. Most of the previous works show that Casson model is the most reliable equation for analyzing the blood behavior in mathematical way of study. But the importance of peripheral layer on blood-like liquid flow has been mentioned by few authors while studying resistance to flow, wall shear stress, plug core radius etc. Again, the study of dispersion with the effect of absorption has lots of physiological relevance and so, in this present paper, we established the effect of peripheral layer on dispersion process in addition with absorption effect and plug core radius.

The exchange coefficient is independent on both peripheral layer and yield stress but the convection coefficient increases with absorption in the present model which is more than a Casson liquid model and less than the single layer Newtonian liquid. The effective diffusion coefficient decreases with absorption, but for small absorption this decrement is less than corresponding values of Casson model, however, more than the single layer Newtonian liquid. Therefore one can conclude 

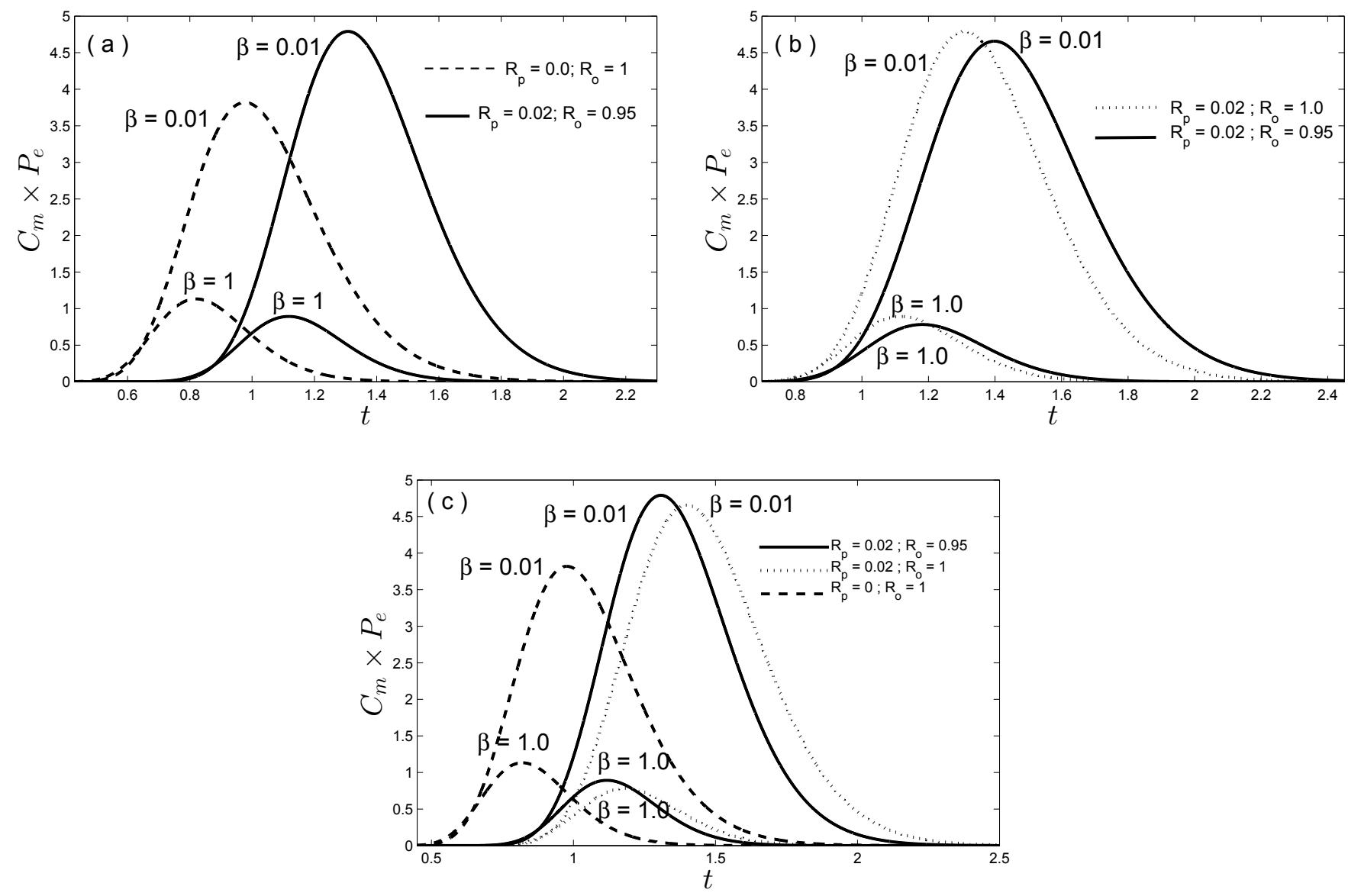

Fig. 7 Variation of mean concentration distribution with time for different values of $R_{p}, R_{o}$ and $\beta$, when $D_{p}=D_{c}=1$.

that the presence of peripheral layer is of major physiological significance relating to the functioning of the blood-like liquid stream, when solute will inject on it. These perceptions agree well with those of existing results as said above and henceforth the applicability of the present model is very much approved. It is believed that this model would definitely be of extensive interest for the physiological issues of blood-like liquid flow.

\section{Acknowledgement}

The first author is thankful to NIT Agartala whereas the third author is thankful to MHRD, India for financial support to pursue their Ph.D. We thank the referees for useful comments that refined the paper to the present form.

\section{References}

[1] Taylor, G. "Dispersion of soluble matter in solvent owing slowly through a tube." Proceedings of the Royal Society A: Mathematical, Physical and Engineering Sciences. 219(1137), pp. 186-203. 1953.

https://doi.org/10.1098/rspa.1953.0139

[2] Aris, R. "On the dispersion of a solute in a fluid owing through a tube." Proceedings of the Royal Society A: mathematical, physical and engineering sciences. 235(1200), pp. 67-77. 1956.

https://doi.org/10.1098/rspa.1956.0065

[3] Sankarasubramanian, R., Gill, W. N. "Unsteady convective diffusion with interphase mass transfer." Proceedings of the Royal Society A: Mathematical, Physical and Engineering Sciences. 333(1592), pp. 115-132. 1973. https://doi.org/10.1098/rspa.1973.0051
[4] Aris, R. "On the dispersion of a solute in pulsating flow through a tube." Proceedings of the Royal Society A: Mathematical, Physical and Engineering Sciences. 259(1298), pp. 370-376. 1960.

https://doi.org/10.1098/rspa.1960.0231

[5] Bandyopadhyay, S., Mazumder, B. S. "On contaminant dispersion in unsteady generalised couette flow." International Journal of Engineering Science. 37(11), pp. 1407-1423. 1999.

https://doi.org/10.1016/S0020-7225(98)00132-3

[6] Paul, S. "Effect of wall oscillation on dispersion in axi-symmetric ows between two coaxial cylinders." ZAMM-Journal of Applied Mathematics and Mechanics/Zeitschrift für Angewandte Mathematik und Mechanik 91(1), pp. 23-37. 2011.

https://doi.org/10.1002/zamm.200700106

[7] Bandyopadhyay, S., Mazumder, B. S. "Unsteady convective diffusion in a pulsatile flow through a channel." Acta Mechanica. 134(1), pp. 1-16. 1999. https://doi.org/10.1007/BF01170300

[8] Sarkar, A., Jayaraman, G. "The effect of wall absorption on dispersion in oscillatory flow in an annulus: application to a catheterized artery." Acta Mechanica. 172(3), pp. 151-167. 2004.

https://doi.org/10.1007/s00707-004-0157-3

[9] Mazumder, B. S., Mondal, K. K. "On solute transport in oscillatory ow through an annular pipe with a reactive wall and its application to a catheterized artery." The Quarterly Journal of Mechanics and Applied Mathematics. 58(3), pp. 349-365. 2005.

https://doi.org/10.1093/qjmam/hbi009

[10] Sharp, M. K. "Shear-augmented dispersion in non-newtonian fluids." Annals of Biomedical Engineering. 21(4), pp. 407-415. 1993.

https://doi.org/10.1007/BF02368633 
[11] Agrawal, S., Jayaraman, G. "Numerical simulation of dispersion in the flow of power law fluids in curved tubes." Applied Mathematical Modelling. 18(9), pp. 504-512. 1994.

https://doi.org/10.1016/0307-904X(94)90329-8

[12] Dash, R. K., Jayaraman, G., Mehta, K. N. "Shear augmented dispersion of a solute in a casson fluid owing in a conduit." Annals of Biomedical Engineering. 28(4), pp. 373-385. 2000.

https://doi.org/10.1114/1.287

[13] Nagarani, P., Sarojamma, G., Jayaraman, G. "Exact analysis of unsteady convective diffusion in casson uid ow in an annulus-application to catheterized artery." Acta Mechanica. 187(1), pp. 189-202. 2006. https://doi.org/10.1007/s00707-006-0316-9

[14] Scott Blair, G. W. "An equation for the ow of blood, plasma and serum through glass capillaries." Nature. 183, pp. 613-614. 1959. https://doi.org/10.1038/183613a0

[15] Charm, S., Kurland, G. "Viscometry of human blood for shear rates of 0-100,000 $\mathrm{sec}^{-1}$." Nature. 206, pp. 617-618. 1965. https://doi.org/10.1038/206617a0

[16] Bugliarello, G., Sevilla, J. "Velocity distribution and other characteristics of steady and pulsatile blood flow in fine glass tubes." Biorheology. 7(2), pp. 85-107. 1970.

[17] Cokelet, G. R. "The rheology of human blood." Biomechanics: Its Foundations and Objectives 72, pp. 63-103. 1972.

[18] Tu, C., Deville, M. "Pulsatile flow of nonnewtonian fluids through arterial stenoses." Journal of biomechanics. 29(7), pp. 899-908. 1996. https://doi.org/10.1016/0021-9290(95)00151-4
[19] Long, Q., Xu, X. Y., Ramnarine, K. V., Hoskins, P. "Numerical investigation of physiologically realistic pulsatile flow through arterial stenosis." Journal of Biomechanics. 34(10), pp. 1229-1242. 2001. https://doi.org/10.1016/S0021-9290(01)00100-2

[20] Srivastava, V. P., Saxena, M. "Two-layered model of casson uid ow through stenotic blood vessels: applications to the cardiovascular system." Journal of Biomechanics. 27(7), pp. 921-928. 1994. https://doi.org/10.1016/0021-9290(94)90264-X

[21] Sankar, D. S. "A two-uid model for pulsatile flow in catheterized blood vessels." International Journal of Non-Linear Mechanics. 44(4), pp. 337-351. 2009. https://doi.org/10.1016/j.ijnonlinmec.2008.12.008

[22] Shukla, J. B., Parihar, R. S. "Dispersion through a flowing fluid with variable viscosity and diffusion coefficient." Physics of Fluids. 23(6), pp. 1271-1273. https://doi.org/10.1063/1.863125

[23] Shukla, J. B., Gupta, S. P. "Dispersion in a bingham plastic fluid: Effects of peripheral layer." Rheologica Acta. 21(6), pp. 713-719. 1982. https://doi.org/10.1007/BF01524515

[24] Sankar, D. S., Lee, U. "Two-fluid casson model for pulsatile blood flow through stenosed arteries: a theoretical model." Communications in Nonlinear Science and Numerical Simulation. 15(8), pp. 2086-2097. 2010. https://doi.org/10.1016/j.cnsns.2009.08.021T 